\title{
Effects of COVID-19 outbreak on the sound environment of the city of Milan, Italy
}

https://doi.org/10.1515/noise-2021-0009

Received Sep 18, 2020; accepted Feb 15, 2021

\begin{abstract}
In this paper, we present the results of the noise monitoring in the urban area of Milan, Italy, associated with COVID-19 outbreak. In order to limit the diffusion of the pandemic, Italy adopted growing levels of restrictive measures, which ended with a 41-day lockdown from March 23 to May 3 2020. This action created a new kind of environment including a remarkable reduction of air, water and soil pollutants, together with a partial recovery of the ecosystems otherwise compromised. The presence of a permanent noise monitoring system made of 24 sensors installed in the city of Milan, allowed capturing the changes in the urban sound environment in a pre, during and post-lockdown period (months from February to June 2020). The comparison of the noise levels in terms of both absolute noise levels (Lden) and hourly noise profiles (median over lockdown period) with the same period of 2019, showed a dramatic reduction of the noise levels of approximately $6 \mathrm{~dB}$. Splitting the contribution of all-non-traffic noises or anomalous noise events (ANE) from the recorded noise levels, we observe a greater contribution of the latter during the lockdown period. The use of DYNAMAP noise prediction system over an extended urban area allowed the evaluation of the population exposure to noise, estimating an increase of $14.9 \%$ and $16.4 \%$ of the exposed population to noises with Lden below $53 \mathrm{~dB}(\mathrm{~A})$ and Lnight below $45 \mathrm{~dB}(\mathrm{~A})$ with respect to 2019 .
\end{abstract}

Keywords: sound environment, COVID-19 pandemic, noise pollution, DYNAMAP project

\footnotetext{
^Corresponding Author: Giovanni Zambon: Dipartimento di Scienze dell'Ambiente e del Territorio e di Scienze della Terra (DISAT), Università di Milano-Bicocca, Piazza della Scienza 1, 20126 Milano, Italy; Email: giovanni.zambon@unimib.it

Chiara Confalonieri, Fabio Angelini, Roberto Benocci: Dipartimento di Scienze dell'Ambiente e del Territorio e di Scienze della Terra (DISAT), Università di Milano-Bicocca, Piazza della Scienza 1, 20126 Milano, Italy
}

ə Open Access. () 2021 G. Zambon et al., published by De Gruyter. (cc) BY License

\section{Introduction}

The unprecedented pandemic outbreak of COVID-19, originated at the end of 2019, forced people to experience a completely new lifestyle. The new working modality and the imposition of social distancing produced implications on people health and wellbeing. Italy's lockdown occurred on 10 March 2020, with the consequence that all non-essential commercial activities, businesses and industries were prohibited, forcing people to "stay home".

Table 1 shows the time line of the Italian Government measures in reply to COVID-19 outbreak. These measures consisted in restrictions, advices and alerts with different level of seriousness.

The lockdown was partially revoked starting from 4 May 2020 and activities slowly returned to normality. On 18 May 2020 and 25 May 2020, we got two further relaxation of restrictions and, finally, in June a complete reopening of all activities though remote working was encouraged to continue.

The lockdown produced a tremendous impact on the economy and time will be needed together with political efforts and huge public investments in those sectors and markets mostly hit by the crisis, to get back to pre-COVID conditions. On the other hand, the lockdown also had a "beneficial" counter effect which reflected positively on air quality and sound environment, owing to a dramatic reduction of air and road traffic volumes. Thus, a completely new sound environment was experienced in all cities [1-4].

The acoustic community organised engaging public organisations and academics to collect noise data [5-7]. This has been achieved through pre-existing noise monitoring networks and on-going measurements.

In particular, the uniqueness in the Italian framework of the permanent noise monitoring network installed in a pilot area of the city of Milan and the motorway surrounding Rome, allowed to capture the changes in the environmental noise conditions engendered by lockdown measures. These permanent noise monitoring networks were implemented in the context of DYNAMAP Life+ European project [8].

In the following sections, a short description of the DYNAMAP noise monitoring network in Milan is given, fol- 
lowed by detailed information on the traffic noise behaviour in a timeframe ranging from January to June 2020 and com- pared to the values recorded in the same period of 2019 in the city of Milan.

Table 1: Phases and measures taken by the Italian Government, through the emanation of DPCMs (Decree of the President of the Council of Ministers), in reply to COVID-19 pandemic

\section{PHASE 1}

Action 1: DPCM 23/02/2020 - effect: schools closed in Lombardia (Region)

Action 2: DPCM 01/03/2020 - effect: manifestations suspended, restaurant and entertainment venues partially closed, partial limitation of work activities

Action 3: DPCM 08/03/2020 - effect: restaurant and entertainment venues closed, banned hangouts, greater limitation of work activities

\begin{tabular}{ll}
\hline \hline LOCK-DOWN \\
\hline Action 4: & $\begin{array}{l}\text { DPCM 22/03/2020 - effect: total limitation of work activities, obligation to stay at home (except for basic } \\
\text { necessities) }\end{array}$ \\
\hline \hline
\end{tabular}

\section{PHASE 2}

Action 5: $\quad$ DPCM 03/05/2020 - effect: first partial reopening of work activities

Action 6: $\quad$ DPCM 17/05/2020 - effect: second partial reopening of work activities

Action 7: $\quad$ DPCM 03/06/2020 - effect: third partial reopening of work activities and displacements between regions

PHASE 3

Action 8: $\quad$ DPCM 15/06/2020 - effect: fourth partial reopening (no schools and public offices)

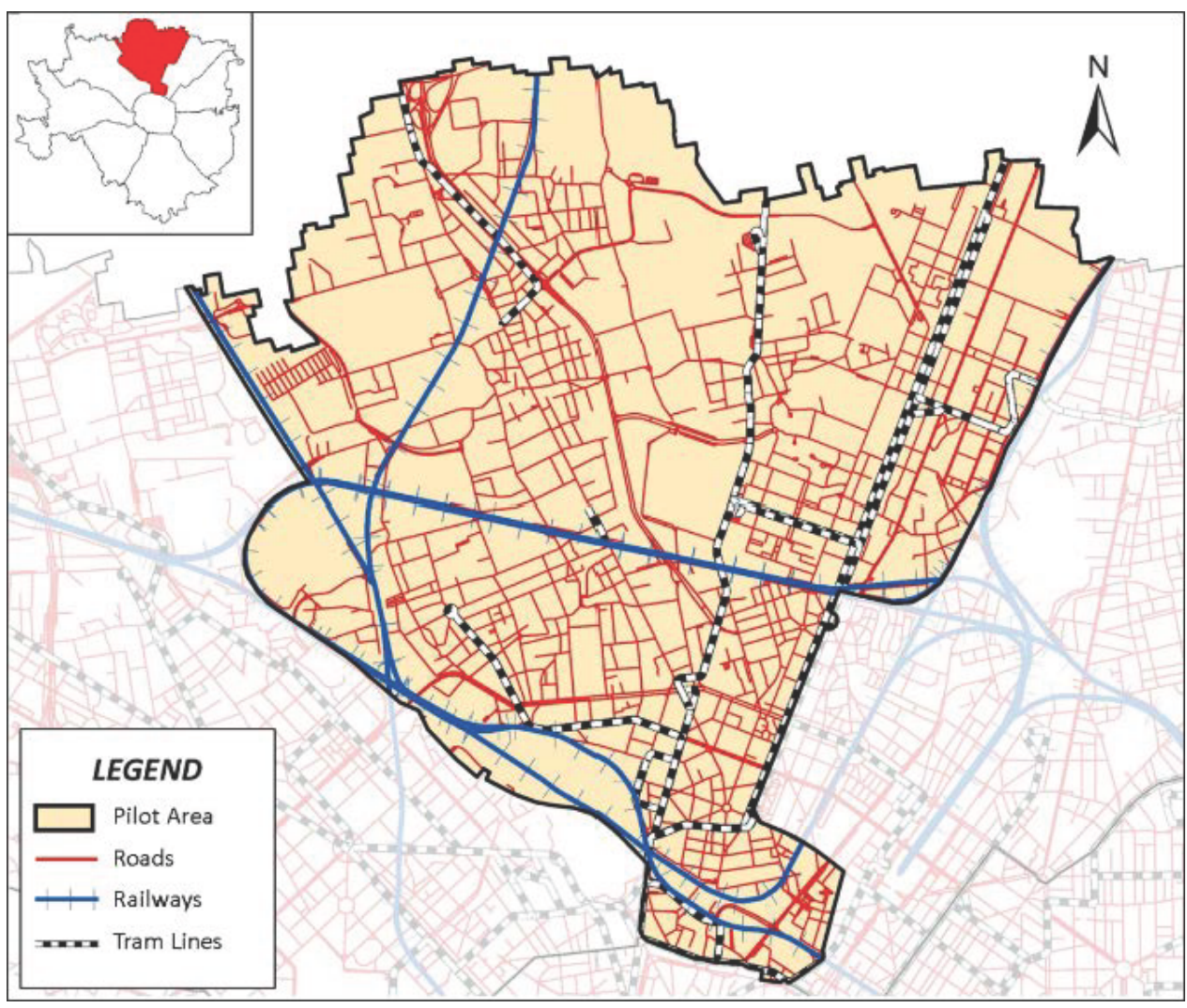

Figure 1: Zone 9 is the pilot area of the city of Milan chosen for the implementation of DYNAMAP system 


\section{DYNAMAP project}

The DYNamic Acoustic MAPping (DYNAMAP) is a LIFE+ project aimed at developing a dynamic noise mapping system able to detect and represent in real time the acoustic impact of road infrastructures in urban and suburban areas through a direct link with a limited number of noise monitoring sensors, permanently installed in the area of study. Zone 9, in the city of Milan, is one of the two pilot areas chosen for the implementation of the system [9] (Figure 1). The other pilot area is in a suburban area of the city of Rome [10]. In the case of Milan, the DYNAMAP network consists of 24 monitoring sensors, which have been appropriately distributed over Zone 9. The sensors are distributed over an area of $21.12 \mathrm{~km}^{2}$ with a population of about 184000 inhabitants, whereas the pilot area of Rome is located along the $68 \mathrm{~km}$ long Motorway A90 that encircles the city and skirting many suburban areas. The presence of these networks allows not only the continuous supply of data for updating dynamic noise maps, but also represent a permanent noise monitoring grid over the city. In the following, we present some of the features of DYNAMAP network.

\subsection{Noise monitoring network}

In both pilot areas, the monitoring network is made of lowcost noise monitoring stations. The sensors are light, small, equipped with a quick coupling system with some removable parts in case of replacement (maintenance) and economical. The control units are installed on the facade of public buildings at a height of about 4 meters, or in the case of impediments due to the presence of architectural constraints and/or high distances from the road, the device, to maintain the established height, is installed on a pole anchored to a gate. The sensors (see an example in Figure 2) consist of a rectangular box measuring $15 \times 11 \times$ $7 \mathrm{~cm}$ and a microphone ( $1 / 4$ inch NEMS microphone) with wind protection. The box contains the acquisition system, the data transmission system and the control electronics.

Other sensor characteristics are listed below:

- 40-100 dB(A) broadband linearity range

- 35-115 dB working range with acceptable THD and narrow-band floor noise level

- $1 \mathrm{~s}$ time base Leq(A) level

- Possibility of audio recording

- Internal circular backup data storage of calculated data

- VPN connection

- GPRS/3G/Wi-Fi connection

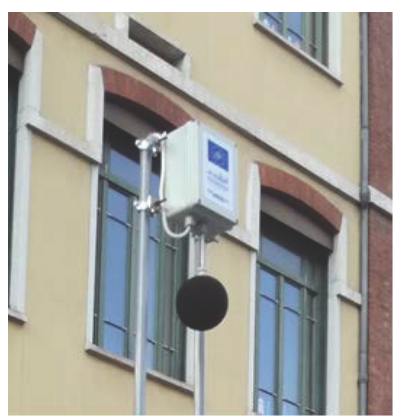

Figure 2: Example of low-cost permanent noise monitoring station of DYNAMAP network

The monitoring stations are installed at relevant receiver locations where sound pressure levels are characterized by the predominance of a single noise source (e.g. vehicular traffic) on the others. For each sensor, a coded output is recorded every second (an example is shown below):

$$
00001-151021113200-58.0-0
$$

The first set of figures is the sensor identifier, the second is a timestamp, the third is the Leq(1s) level in $\mathrm{dB}(\mathrm{A})$ units and the fourth one is an indicator standing for "traffic" $(0)$ or "non-traffic"(1) event (Anomalous Noise Event Algorithm, ANED, used for data "cleaning", is a built-in filter; see Sect. 2.2 for details). All data are stored at a frequency of $1 \mathrm{~Hz}$ in a remote web server through GPRS/3G wireless data communication system. In this way, it is possible to recall and view historical data over long periods. Levels without Anomalous Noise Events (ANEs) can be post-processed for calculating Leq levels at different time scales.

\subsection{Filtering of Anomalous Noise Events (ANED algorithm)}

Usually, acoustic maps are computed by considering the vehicle flows averaged over long periods of time (e.g., one year), which need to be validated by means of measurements. For this purpose, the original acoustic data has to be filtered by removing non-traffic events (e.g. sirens, horns, airplane flyovers, noisy human activities, technical facilities, etc.) since their presence can bias the A-weighted equivalent sound level (LAeq). An Anomalous Noise Events Detector (ANED) algorithm, developed by the University La Salle of Barcelona, operates on audio recordings obtained from low-cost control units and distinguishes acoustic events not related to road traffic from noises from vehicular traffic (RTN, Road Traffic Noise) [11, 12]. 


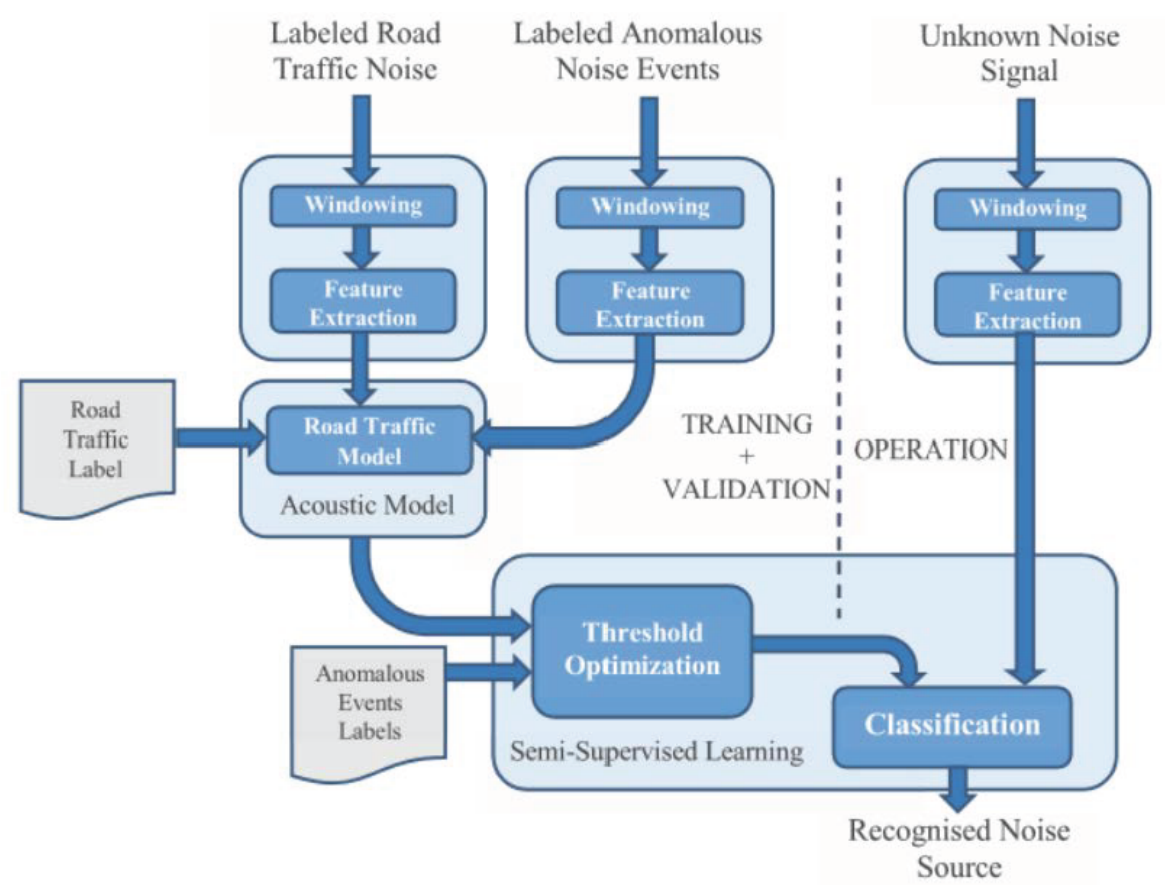

Figure 3: Block diagram of the ANED algorithm

Low-cost sensors apply the distinction between ANE and RTN on the basis of the spectral components and send this information to the web server hosting a GIS software (a software tool based in a GIS platform that performs real time noise maps updating) together with the equivalent noise level (LAeq,1s). At this point, the noise levels measured and labeled by the algorithm as RTN will be used to update the noise level in real time; while those labeled as ANE, will not be considered (see block diagram in Figure 3). Removing a specific anomalous event from the time history can change the LAeq of the time interval considered, making the representation on the map more realistic of the actual road traffic noise.

The ANED algorithm allows the evaluation of anomalous events, filtering acoustic data by removing non-traffic events and it has been used to support the analysis, identifying the contribution of the different typologies of noise sources (anthropogenic sources, natural sounds, etc.).

\subsection{Dynamic acoustic map}

DYNAMAP's calculation scheme is based on the idea that roads making up the urban network display similar traffic noise behaviour so that one can group and describe them by a single noise map [9, 12]. Each map thus represents a group of roads with similar noise profiles (same dynamic behaviour). To achieve this goal, we performed a detailed analysis of traffic noise data, recorded from 93 sites distributed all over the urban area of the city of Milan. A statistical analysis allowed identifying groups of road segments sharing the same noise behaviour. This behaviour was found to be driven by a non-acoustic parameter associated with the logarithm of the total traffic low $(x=\log (T T))$. The knowledge of this parameter allowed the extension of the results to all non-monitored roads and therefore their association with a specific group (noise map). Each static noise map is the result of CADNA commercial software calculation. DYNAMAP has been implemented in a pilot area called Zone 9, made of about 2000 road stretches, which, for convenience, have been divided into six groups, each one represented by a noise map (six static maps).

The dynamic character of each static noise map is obtained by adding the contribution of the 24 monitoring stations, four in each group [9] (Figure 4).

Operatively, after filtering the anomalous noise events by applying ANED [11], for each sensor we evaluate a level variation between the reference value (evaluated from the static acoustic map at the point corresponding to the position of the monitoring station) and the measured level (at sensor site). We calculate the mean variation for each group and add them to each static noise map. Eventually, the global noise map is obtained by properly adding each updated noise map (see working scheme in Figure 5).

We decided to update DYNAMAP according to the following scheme: 

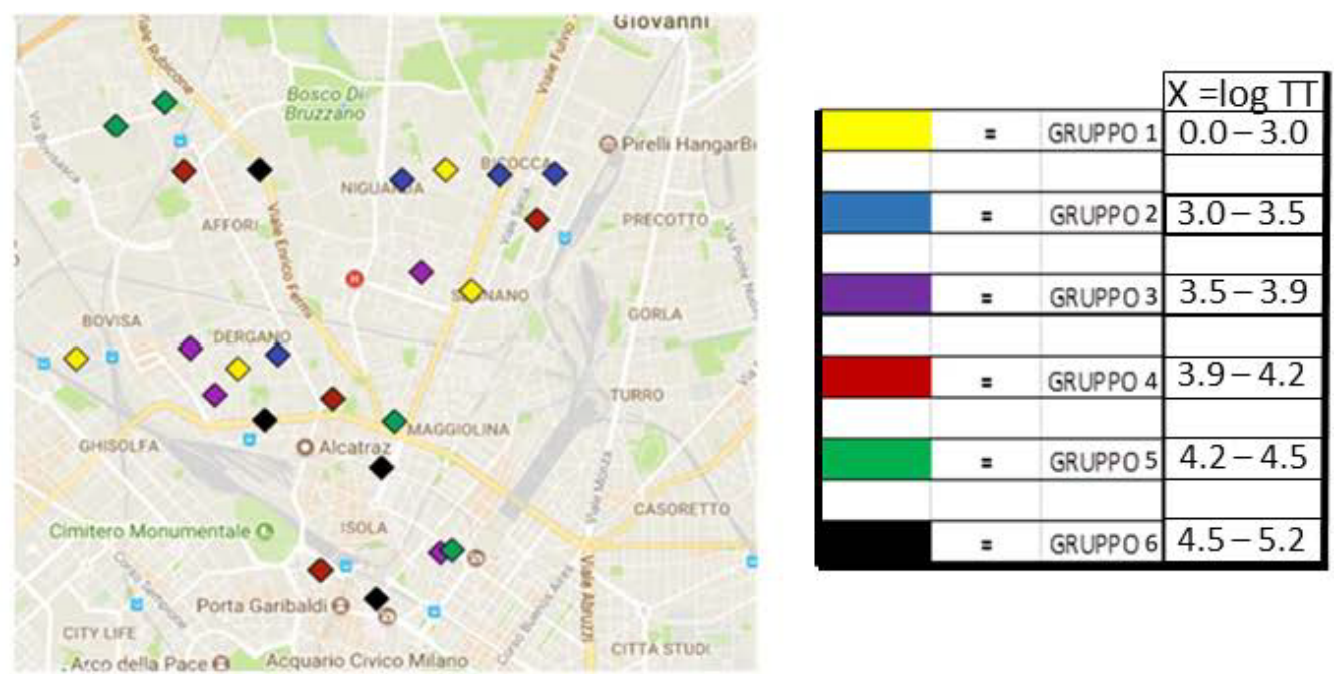

Figure 4: Distribution of the 24 sensors in the pilot area of Milan split into each group represented by different colours. The table on the left reports the value of the non-acoustic parameter, $x=\log (T T)$, calculated for each group

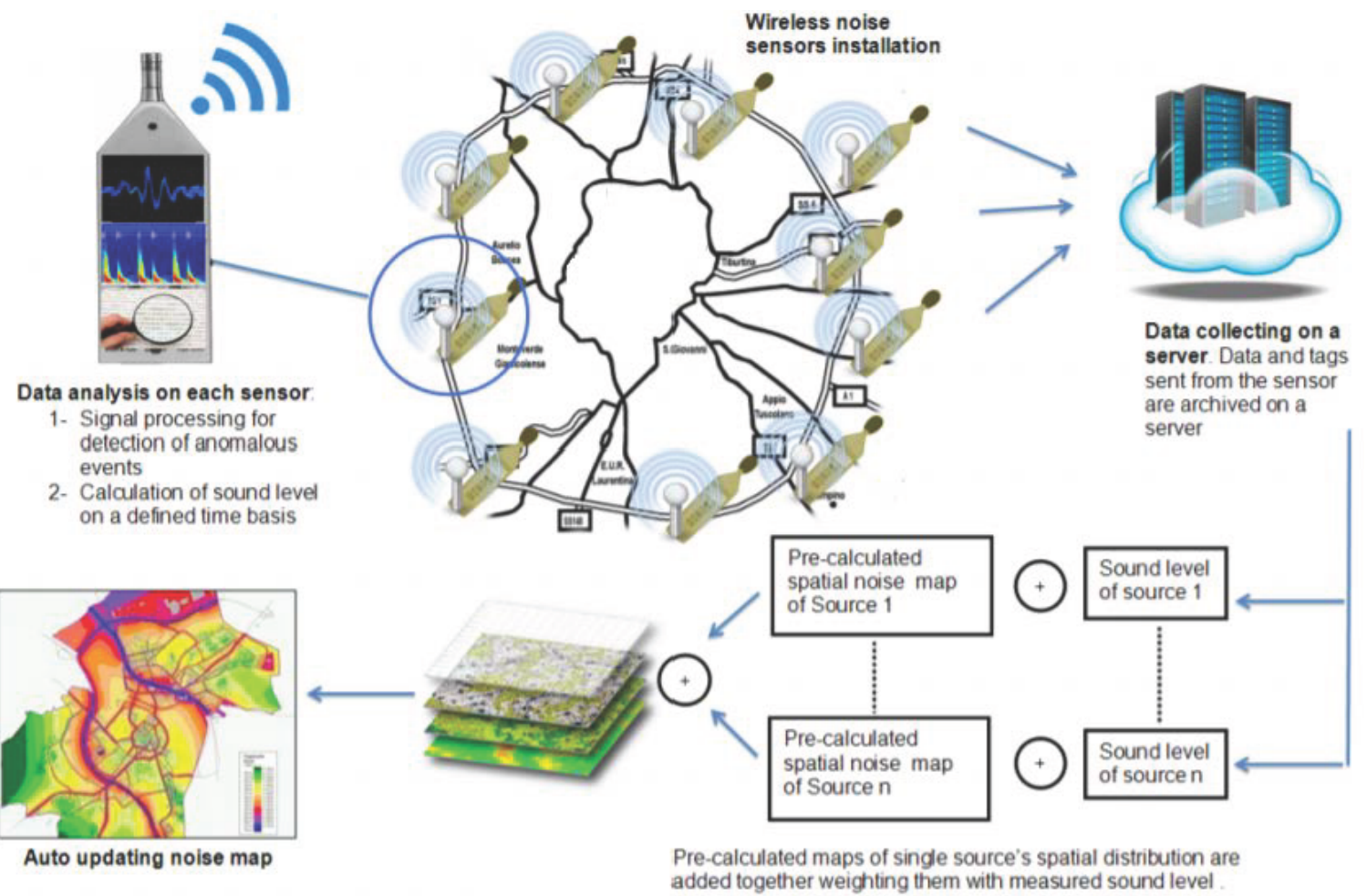

Figure 5: DYNAMAP working scheme

$$
\begin{aligned}
& \tau=5 \min \text { for }(07: 00-21: 00) \\
& \tau=15 \min \text { for }(21: 00-01: 00)
\end{aligned}
$$$$
\tau=60 \text { min for (01:00-07:00) }
$$

Details about the calculation procedure, method and accuracy of DYNAMAP can be found in refs [9].

The Dynamic noise maps are available on the official DYNAMAP webpage [8].

\section{Data collection from the monitoring network}

The entire DYNAMAP network made of 24 monitoring stations has been used to investigate the noise level changes generated by the different sound sources over the studied period. In addition to this, we can count on a database of 


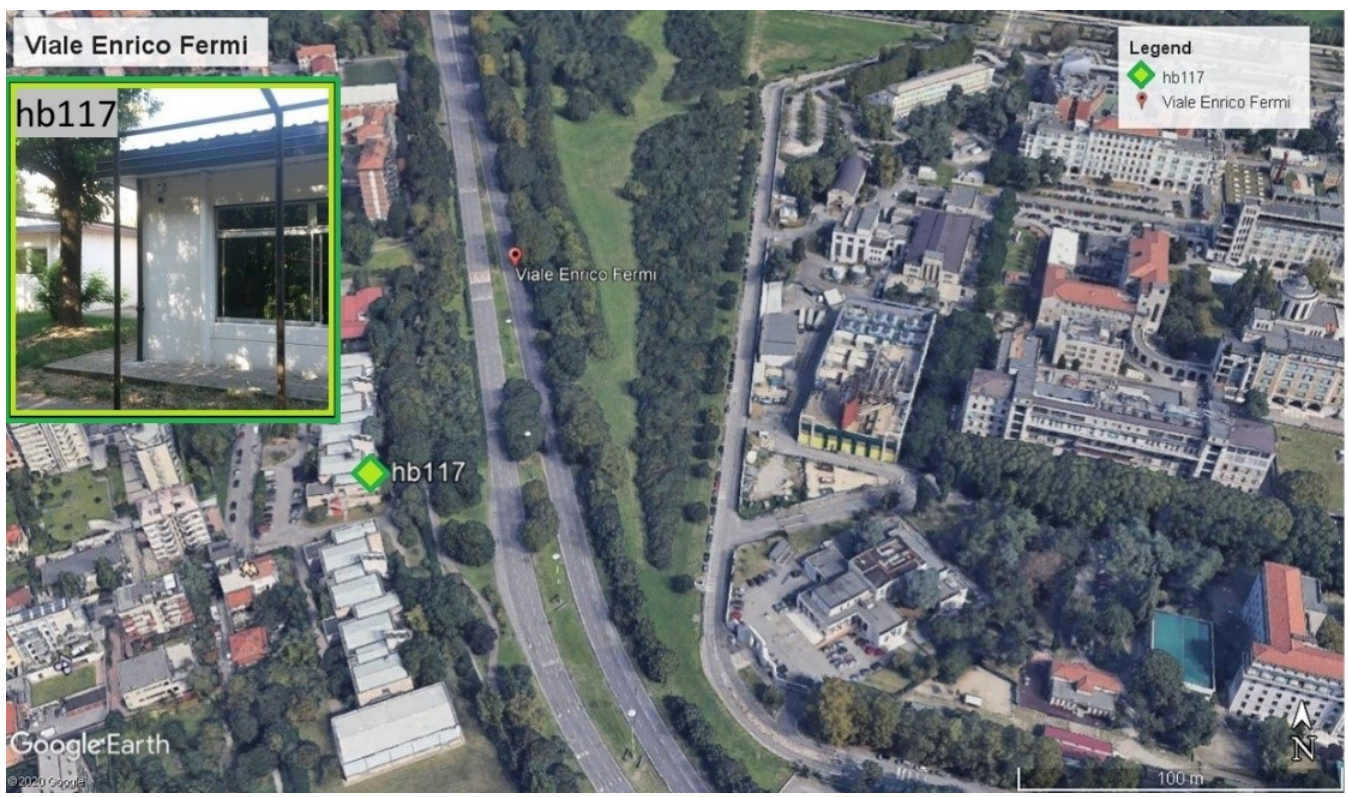

Figure 6: Google Earth's view illustrating the position of hb 117 sensor

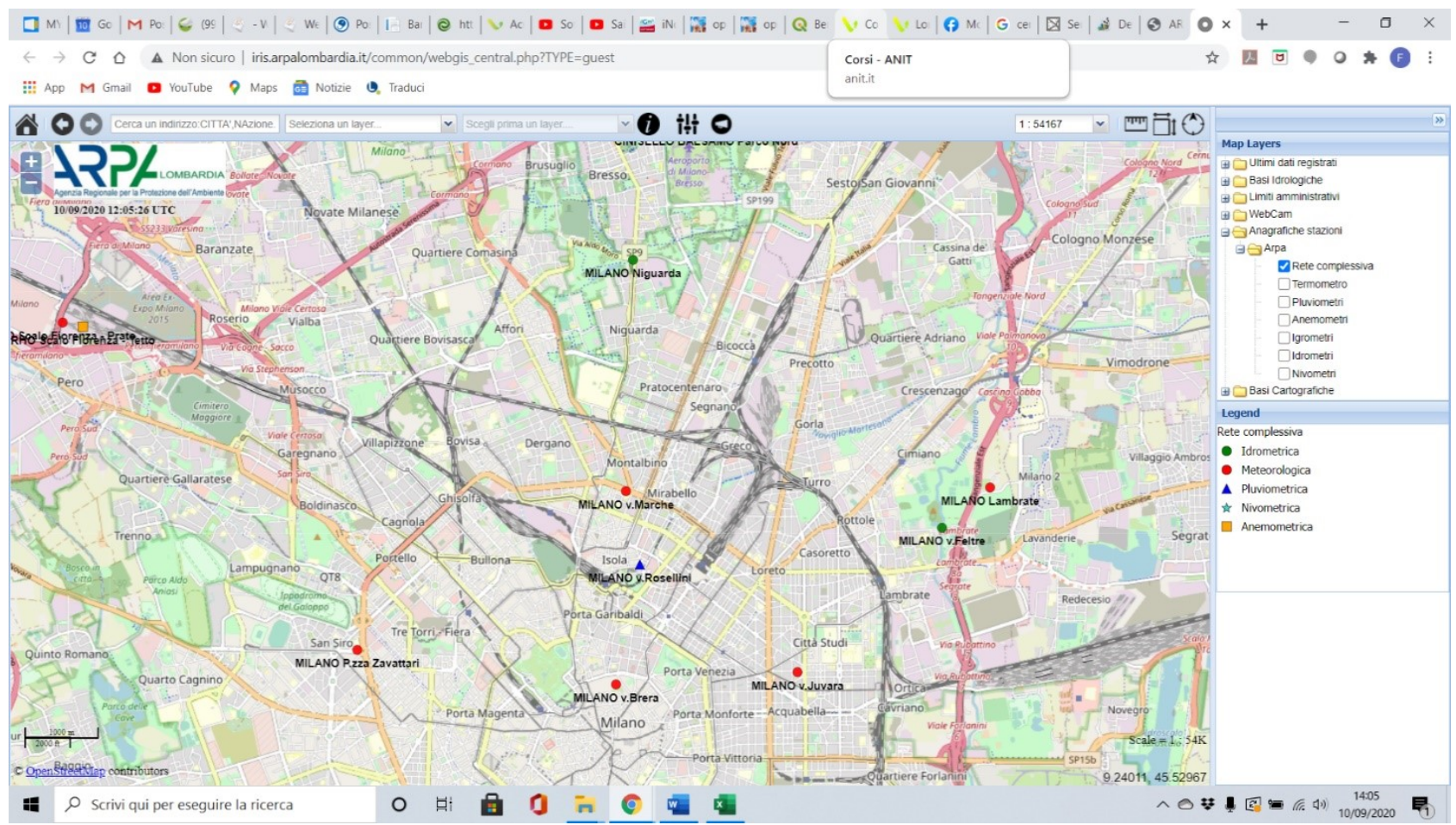

Figure 7: ARPA Lombardia weather stations considered for the removal of noise data for days with unfavorable weather condition

acoustic measurements recorded since August 2017 (date of DYNAMAP operability). This is particularly important considering the aim of this research to compare the noise levels recorded during the lockdown period and the same period of 2019.

DYNAMAP returns the noise levels in two shapes: $\mathrm{cu}-$ mulative or raw data (in which all types of noise sources are considered) and purged or cleaned data (in which only the contribution of RTN is retained) [11]. The analysis has been carried out considering both cumulative and "cleansed" data for all 24 survey sites. As an example, the results obtained for a significant site near a thoroughfare road (Viale Enrico Fermi) in the proximity of Niguarda hospital (Figure 6) is reported. 
In the following, we present the scheme followed for the analysis of the data recorded from each of the 24 monitoring stations:

- Period considered

Period analyzed: 1 January-21 June 2020

Reference period: 1 January-21 June 2019

Noise indicators: Lden total, Lden upon ANED filtering (Sect. 2.2).

- Data treatment

Identification of missing data due to sensor off-line or temporary out of use

Removal of noise data for days with unfavorable weather (rain rate $>2 \mathrm{~mm} / \mathrm{h}$ and wind speed $>5 \mathrm{~m} / \mathrm{sec}$ ) obtained by cross-checking ARPA (Regional Agency for Environmental Protection) Lombardia weather stations (Figure 7 illustrates the position of the reference weather stations).

The recorded noise data refer to the periods corresponding to the Italian Government measures in reply to COVID-19 pandemic according to the time-line reported in Table 1.

\section{Results and discussion}

\subsection{Example of analysis of a specific site}

By way of illustration, we report the results of the analysis carried out on the monitoring station hb 117 installed in the proximity of Viale Enrico Fermi (200 m from Niguarda Hospital), an arterial thoroughfare characterized high capacity traffic flows (see Figure 6). This site is mainly residential, with the presence of sensitive buildings (it is located a few hundred meters from Niguarda Hospital and school sites). Near the area there are small playgrounds, and an important and vast urban park (Parco Nord). In addition to via Enrico Fermi, the area is crossed by numerous local and neighborhood streets, mainly serving the residences in the area. There are not noisy sites, such as industries, but there is an open air market (organized weekly during the preCovid period), as well as some commercial activities, such as retail shops and restaurants.

Figure 8 illustrates the total Lden trend (including ANEs) over the period 1 January and 21 June 2020, that is the period between the initial diffusion of COVID-19 in Italy, the lockdown period and the initial reopening of all activities. As it can be observed, the noise level trend follows the impact of human activity and of vehicular flows:

- pre-lockdown: presence of stationary levels

- lockdown period: noise levels at minimum

- post-lockdown period: gradual increase of noise levels

Another observed occurrence regards the social consequences of the entry into force of the restrictive regulation lately named as 'fear-of-running-out'. The day after the first four Italian Government measures, we can observe, contrary to what expected, an unusual increase of the noise levels. These are most likely due to pantry stockpiling as

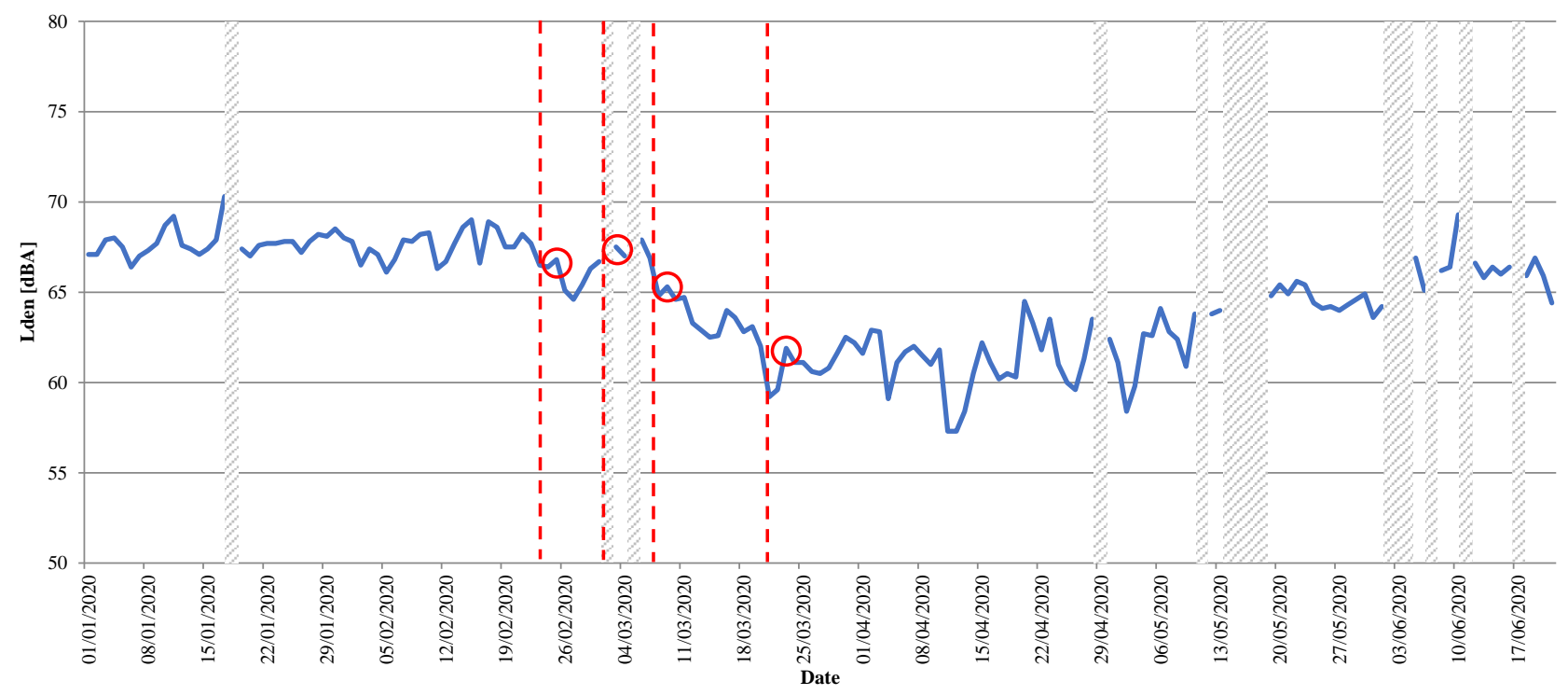

Figure 8: Time evolution of Lden levels in the period between 1 January and 21 June 2020 recorded at the noise monitoring sensor hb117 (Viale E. Fermi). In the figure, the white bands refer to non-available data, the dashed red lines to the dates of entry into force of restrictive regulations and the circles the 'fear-of-running-out' Lden level hikes 


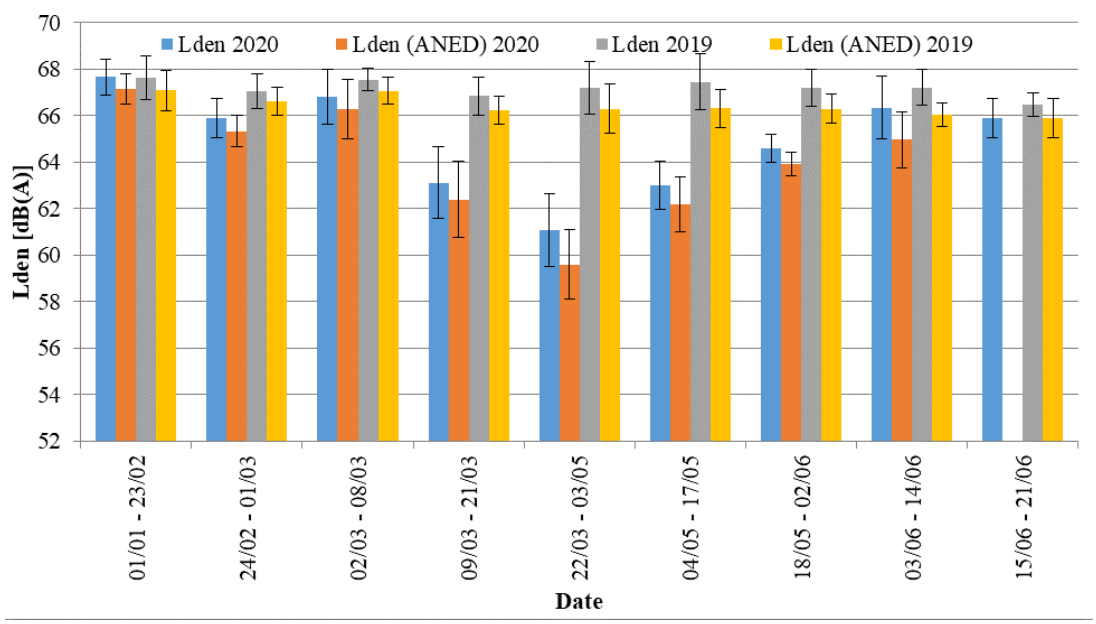

Figure 9: Time evolution of Lden levels in the period from 1 January and 21 June 2019 and 2020 recorded at the noise monitoring sensor hb 117 (Viale E. Fermi). Each period corresponds to the dates of the different Italian Government measures reported in Table 2. In the figure the total Lden and Lden (ANEs) levels are also shown with their standard deviations. For the period 15/06 - 21/06, Lden (ANED) data for 2020 are not available due to the detected anomalous functioning of the ANED algorithm

Table 2: Time intervals corresponding to the dates of the different Italian Government measures considered for the analysis of noise levels

\begin{tabular}{lc}
\hline Period & Date \\
\hline $1-$ before first action (normal situation) & $01 / 01 / 2020-23 / 02 / 2020$ \\
2 - between first and second action & $24 / 02 / 2020-01 / 03 / 2020$ \\
3 - between second and third action & $02 / 03 / 2020-08 / 03 / 2020$ \\
$4-$ between third and fourth action & $09 / 03 / 2020-21 / 03 / 2020$ \\
$5-$ between fourth and fifth action & $22 / 03 / 2020-03 / 05 / 2020$ \\
6 - between fifth and sixth action & $04 / 05 / 2020-17 / 05 / 2020$ \\
7 - between sixth and seventh action & $18 / 05 / 2020-02 / 06 / 2020$ \\
$8-$ between seventh and eighth action & $03 / 06 / 2020-14 / 06 / 2020$ \\
$9-$ after eighth action & $15 / 06 / 2020-21 / 06 / 2020$ \\
\hline
\end{tabular}

a reaction to stress and fear of infection, making people react regardless of whether it is rational and leading them to engage in the same behaviour. In Figure 8, the dashed red lines refer to the dates of entry into force of restrictive regulations and the circles the 'fear-of-running-out' Lden level hikes.

In Figure 9, we report the time evolution of Lden levels for the time period between 1 January and 21 June 2020, calculated for the time intervals corresponding to the dates of the different Italian Government measures reported in Table 2. As a reference, the Lden levels corresponding to the same period of 2019 have been added.

Figure 9 also reports the Lden levels corresponding to Lden total (both traffic and non-traffic noise sources) and Lden upon ANED filtering (just RTN sources).

The analysis shows that Lden levels corresponding to 2019 (gray and yellow bars) remain stable over different periods. The first period, from 1 January to 23 February (first health emergency), 2019 and 2020 data are aligned, confirming that, in general, the noise trend profiles are almost stationary, thus suggesting that acoustic-wise 2020, up to 23 February can be considered as "standard". From the end of February, the introduction of limitation measures regarding all non-essential commercial activities, businesses and industries, caused a significant reduction of noise, with minimum levels, reached during the lockdown period (from 23 March to 3 May), of about $6.6 \mathrm{~dB}$ for the total Lden. As for the contribution of just the traffic component, that is the Lden levels filtered from anomalous noise events (ANEs), we can observe that these values are always lower than the total Lden and follow the same trend. Although this might seem an expected result, it means that besides road traffic also the other activities are drastically reduced during lockdown. In particular, the difference between the total Lden levels and those without ANEs during the lockdown is greater (1.5 $\mathrm{dB}$ ) than the one measured in the reference period (2019) 


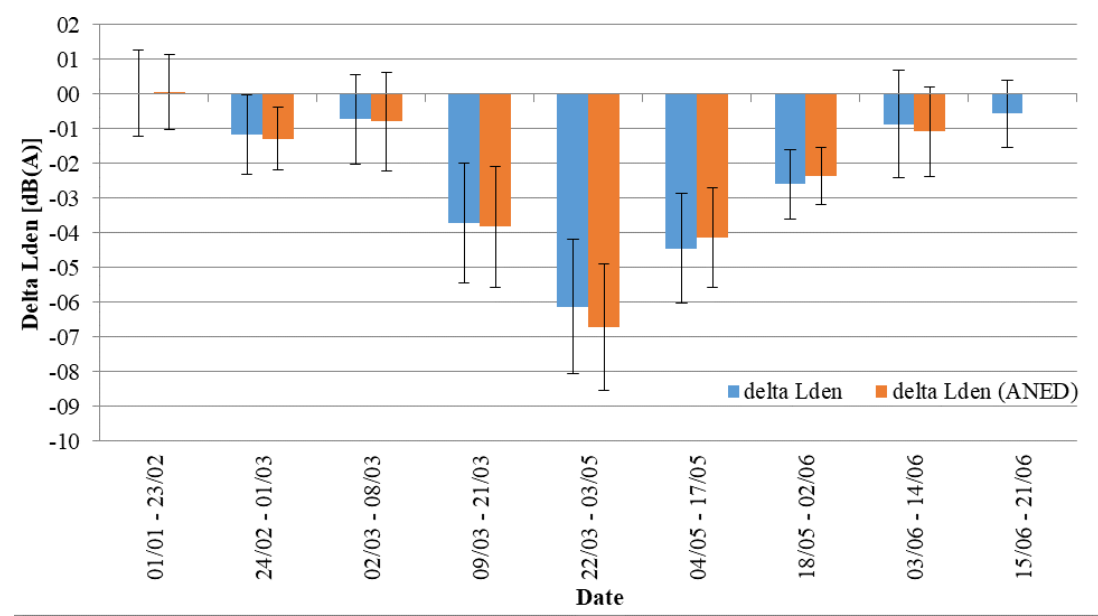

Figure 10: Trend of the differences (delta) between Lden levels for the years 2019 and 2020 for the noise monitoring sensor hb 117 (Viale E. Fermi). The difference is shown for both Lden total and Lden (ANEs) with their standard deviations. Each period corresponds to the dates of the different Italian Government measures reported in Table 2. For the period 15/06 - 21/06, delta Lden (ANED) data are not available due to the unavailability of the Lden (ANED) 2020 data, as we can see in Figure 9

(1.1 dB). This difference can be explained by assuming that in presence of low levels of traffic noise and anthropogenic sources, natural sounds, such as for example birds singing activities, may emerge and cause, in absence of high levels of traffic noise, an increase of the overall Lden value with respect to the filtered one. Another possible cause could be the increase of health emergency calls (transit of ambulances with sirens) recorded by the monitoring station located close to Niguarda hospital (Figure 6). Indeed, more anomalous events have been recorded during the lockdown period due to an increase of the number of interventions by medical aid.

In order to illustrate more closely this concept, Figure 10 reports the trend of the differences (delta) between Lden levels of the years 2019 and 2020, accounting for all noise sources (Lden total) and just the traffic noise contribution (Lden ANEs) referred to the periods shown in Table 2.

The analysis confirms that the noise levels during the pre-lockdown period (from 1 January to 23 February) do not present significant variations with respect to the same 2019 period. As for the noise level reduction over the lockdown period (highest drop), the difference with respect to the reference period is around $6 \mathrm{~dB}$ for Lden total and $6.5 \mathrm{~dB}$ for Lden ANEs. After easing the restrictions (from 4 May), a consistent increase of noise levels can be clearly observed. To be noted, how the difference of Lden (ANEs) levels for the two periods is higher than the corresponding Lden total, especially during the lockdown period.

Another interesting feature is displayed in Figure 11, reporting the hourly median daily profile calculated during lockdown and the same period of 2019 for the monitoring sensor hb 117. The mean noise reduction is of about $6 \mathrm{~dB}$

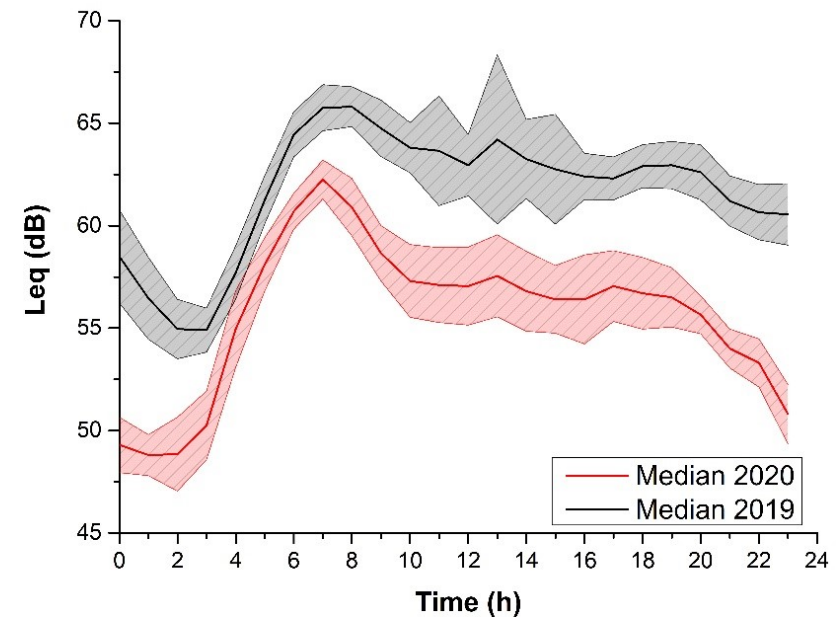

Figure 11: Hourly median daily profile calculated during lockdown and the same period of 2019 for the monitoring sensor hb 117. The coloured bands represent the inter-quartile range

with a minimum of $3.5 \mathrm{~dB}$ at 7:00 and a maximum of $9.8 \mathrm{~dB}$ at 23:00. This result underlines a reduced traffic flow in the morning with the presence of just essential services and activities but with a complete absence of night-life.

\subsection{Analysis of all the noise monitoring network in Milan}

In the following, we report the results of the data analysis for all the noise monitoring stations installed in the city of Milan, for the periods corresponding to the dates of the different Italian Government measures reported in Table 2. 


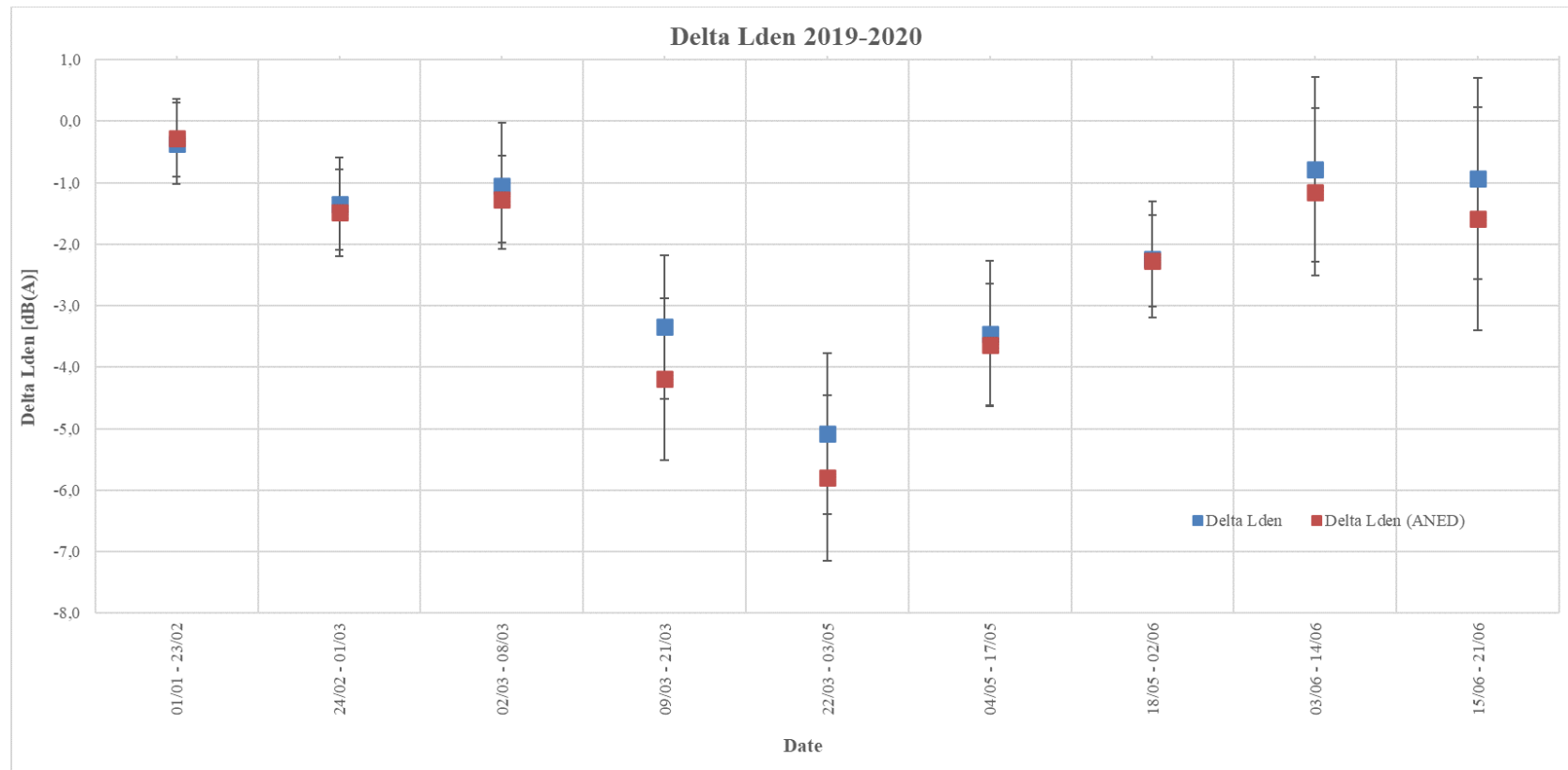

Figure 12: Mean trend of the differences (delta) between Lden levels for the years 2019 and 2020 for all noise monitoring sensors. The difference is shown for both Lden total and Lden (ANEs) with their standard deviations. Each period corresponds to the dates of the different Italian Government measures reported in Table 2

In particular, Figure 12 illustrates the mean trend of the differences (delta) between Lden levels for the years 2019 and 2020 for all noise monitoring sensors. The difference is shown for both Lden total and Lden (ANEs) with their standard deviations.

The data confirm that

a) the pre-lockdown period (from 1 January to 23 February) does not present significant differences with 2019 Lden levels and

b) the mean trends reflect the results observed for the monitoring sensor hb117.

The maximum noise level reduction reached a value of about 5-6 dB during lockdown. In this case, the Lden ANED variations are always higher than the levels referred to Lden total, especially in correspondence of the lockdown period. This observation highlights that the primary urban noise source is vehicular traffic that, during the restriction measures caused by health emergency, dropped. On the contrary, the other non-traffic noise sources were less affected by circumstances such as in the case of natural noises emerging from the background and becoming more significant, or amplified by an increased rate of emergency interventions.

\subsection{Analysis of noise maps: period 2019-2020}

In order to have more insights into the effects produced by the lockdown period over an extended urban area, we harnessed DYNAMAP system capability of predicting acoustic noise maps over the pilot area (named Zone 9) of Milan from a historical archive of data. Figure 13 shows the Lden (ANED) level averaged over the period (23 March-3 May) for the reference year and 2020. The Lden levels are displayed in different colours.

The comparison of the noise maps confirms an average drop of Lden levels of about 5-7 dB.

DYNAMAP allows the noise levels in correspondence to each receptor (building façade) to be calculated and exported; each receptor is also associated with the resident population, thus, with the possibility of evaluating the population exposure to the traffic noise. For the studied area, the total receptors (buildings) are 16530 to which correspond 208027 resident people.

Specifically, considering the source "road traffic", in order to avoid the onset of damages to health and effects on sleep, The World Health Organization (WHO) in the "Environmental noise guidelines for the European Region" [13] strongly recommends ensuring compliance with two noise thresholds, respectively equal to: Lden $53 \mathrm{~dB}(\mathrm{~A})$ and Lnight $45 \mathrm{~dB}(\mathrm{~A})$. 

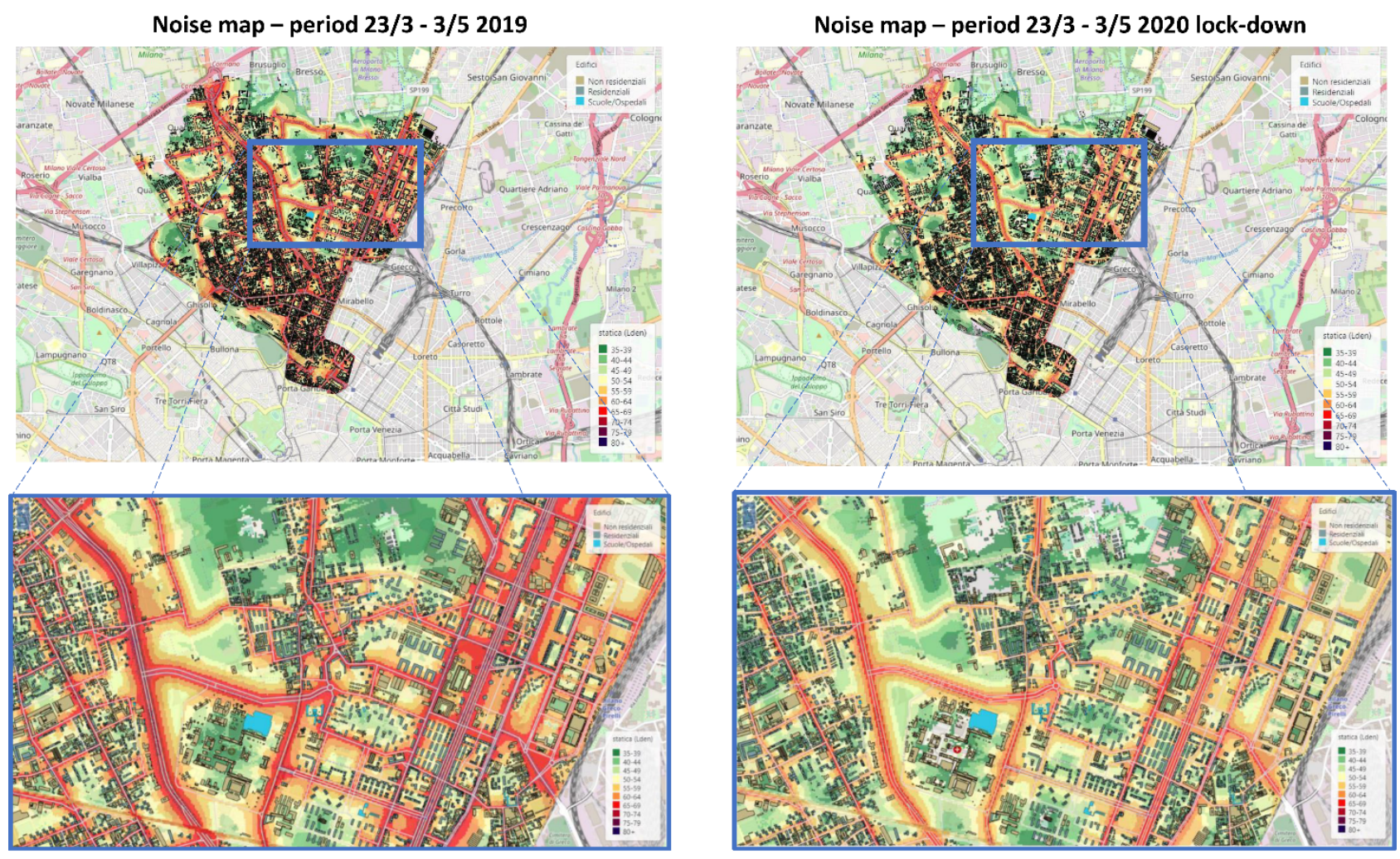

Figure 13: Comparison between 2019 and 2020 noise maps (left upper panel and right upper panel, respectively). The indicator reported is Lden (total or ANED) level averaged over the period (23 March-3 May). Lden levels are displayed in different colors. A zoom of a specific area is illustrated (left lower panel and right lower panel) to appreciate the Lden levels changes

Table 3: Percentage of buildings and population that, during the lockdown period, passed to a noise exposition below $L d e n 53 \mathrm{~dB}(A)$ and Lnight $45 \mathrm{~dB}(\mathrm{~A})$ with respect to 2019

\begin{tabular}{|c|c|c|c|c|c|}
\hline & $\begin{array}{c}\text { Period 23/3 - } \\
31 / 52019\end{array}$ & $\begin{array}{c}\text { Period 23/3- } \\
31 / 52020\end{array}$ & & $\begin{array}{c}\text { Period 23/3 - } \\
31 / 52019\end{array}$ & $\begin{array}{r}\text { Period 23/3- } \\
31 / 52020\end{array}$ \\
\hline $\mathbf{N}^{\circ}$ of buildings & 7224 & 9775 & $\mathbf{N}^{\circ}$ of buildings & 7106 & 9875 \\
\hline Lden $\leq 53 \mathrm{~dB}(\mathrm{~A})$ & & & Lnight $\leq 45 \mathrm{~dB}(\mathrm{~A})$ & & \\
\hline$\%$ of buildings & $43.7 \%$ & $59.1 \%$ & $\%$ of buildings & $43 \%$ & $59.7 \%$ \\
\hline Lden $\leq 53 \mathrm{~dB}(\mathrm{~A})$ & & & Lnight $\leq 45 \mathrm{~dB}(\mathrm{~A})$ & & \\
\hline $\mathbf{N}^{\circ}$ of people & 59234 & 90363 & $\mathbf{N}^{\circ}$ of people & 57903 & 91942 \\
\hline Lden $\leq 53 \mathrm{~dB}(\mathrm{~A})$ & & & Lnight $\leq 45 \mathrm{~dB}(\mathrm{~A})$ & & \\
\hline$\%$ of people & $28.5 \%$ & $43.4 \%$ & $\%$ of people & $27.8 \%$ & $44.2 \%$ \\
\hline Lden $\leq 53 \mathrm{~dB}(\mathrm{~A})$ & & & Lnight $\leq 45 \mathrm{~dB}(\mathrm{~A})$ & & \\
\hline
\end{tabular}

In Table 3, we report the percentage of buildings and population that, during lockdown, passed to a noise exposition below Lden $53 \mathrm{~dB}(\mathrm{~A})$ and Lnight $45 \mathrm{~dB}(\mathrm{~A})$ with respect to 2019. Considering the number of exposed buildings, the increase is $+15.4 \%$ and $+16.7 \%$ for Lden and Lnight, respectively. As for the exposed population, the percentage increase is $+14.9 \%$ and $+16.4 \%$ for Lden and Lnight, respectively.
In Table 4, the variation 2019-2020 of the number of exposed buildings for each $5 \mathrm{~dB}$ interval are also illustrated. To be noted that, the percentage of buildings exposed to Lden noise levels between 73 and $68 \mathrm{~dB}(\mathrm{~A})$ passed from $13.1 \%$ to $1.4 \%$ and from $8.8 \%$ to $30.1 \%$ for noise levels below $43 \mathrm{~dB}(\mathrm{~A})$. A marked reduction has also been observed when referring to Lnight noise levels. In this case, the percentage passed from $13.3 \%$ to $1.4 \%$ in the interval 60 and $65 \mathrm{~dB}(\mathrm{~A})$ and from $24.3 \%$ to $46.4 \%$ for Lnight $<40 \mathrm{~dB}(\mathrm{~A})$. 
Table 4: Variation 2019-2020 of the number of exposed buildings for each $5 \mathrm{~dB}$ interval

\begin{tabular}{|c|c|c|c|c|c|}
\hline & $\begin{array}{c}\text { Period 23/3- } \\
31 / 52019\end{array}$ & $\begin{array}{c}\text { Period 23/3- } \\
31 / 52020\end{array}$ & & $\begin{array}{c}\text { Period 23/3 - } \\
31 / 52019\end{array}$ & $\begin{array}{c}\text { Period 23/3 } \\
31 / 52020\end{array}$ \\
\hline $\begin{array}{l}\mathrm{N}^{\circ} \text { of buildings } \\
\text { Lden } \geq 78 \mathrm{~dB}(\mathrm{~A})\end{array}$ & 0 & 0 & $\begin{array}{l}\mathrm{N}^{\circ} \text { of buildings } \\
\text { Lnight } \geq 75 \mathrm{~dB}(\mathrm{~A})\end{array}$ & 0 & 0 \\
\hline$\%$ of buildings & $0 \%$ & $0 \%$ & $\%$ of buildings & $0 \%$ & $0 \%$ \\
\hline Lden $\geq 78 \mathrm{~dB}(\mathrm{~A})$ & & & Lnight $\geq 75 \mathrm{~dB}(\mathrm{~A})$ & & \\
\hline $\mathbf{N}^{\circ}$ of buildings & 407 & 0 & $\mathbf{N}^{\circ}$ of buildings & 1 & 0 \\
\hline $73 \leq$ Lden $\leq 77.9$ & & & $70 \leq$ Lnight $\leq 74.9$ & & \\
\hline$\%$ of buildings & $2.5 \%$ & $0 \%$ & $\%$ of buildings & $0.1 \%$ & $0 \%$ \\
\hline $73 \leq$ Lden $\leq 77.9$ & & & $70 \leq$ Lnight $\leq 74.9$ & & \\
\hline $\mathbf{N}^{\circ}$ of buildings & 2165 & 231 & $\mathbf{N}^{\circ}$ of buildings & 605 & 0 \\
\hline $68 \leq$ Lden $\leq 72.9$ & & & $65 \leq$ Lnight $\leq 69.9$ & & \\
\hline$\%$ of buildings & $13.1 \%$ & $1.4 \%$ & $\%$ of buildings & $3.7 \%$ & $0 \%$ \\
\hline $68 \leq L$ den $\leq 72.9$ & & & $65 \leq$ Lnight $\leq 69.9$ & & \\
\hline $\mathbf{N}^{\circ}$ of buildings & 2412 & 1791 & $\mathbf{N}^{\circ}$ of buildings & 2200 & 228 \\
\hline $63 \leq$ Lden $\leq 67.9$ & & & $60 \leq$ Lnight $\leq 64.9$ & & \\
\hline$\%$ of buildings & $14.6 \%$ & $10.8 \%$ & $\%$ of buildings & $13.3 \%$ & $1.4 \%$ \\
\hline $63 \leq$ Lden $\leq 67.9$ & & & $60 \leq$ Lnight $\leq 64.9$ & & \\
\hline $\mathbf{N}^{\circ}$ of buildings & 2180 & 2543 & $\mathbf{N}^{\circ}$ of buildings & 2318 & 1675 \\
\hline $58 \leq$ Lden $\leq 62.9$ & & & $55 \leq$ Lnight $\leq 59.9$ & & \\
\hline$\%$ of buildings & $13.2 \%$ & $15.4 \%$ & $\%$ of buildings & $14 \%$ & $10.1 \%$ \\
\hline $58 \leq$ Lden $\leq 62.9$ & & & $55 \leq$ Lnight $\leq 59.9$ & & \\
\hline $\mathbf{N}^{\circ}$ of buildings & 2179 & 2233 & $\mathbf{N}^{\circ}$ of buildings & 2173 & 2533 \\
\hline $53 \leq$ Lden $\leq 57.9$ & & & $50 \leq$ Lnight $\leq 54.9$ & & \\
\hline$\%$ of buildings & $13.2 \%$ & $13.5 \%$ & $\%$ of buildings & $13.1 \%$ & $15.3 \%$ \\
\hline $53 \leq$ Lden $\leq 57.9$ & & & $50 \leq$ Lnight $\leq 54.9$ & & \\
\hline $\mathbf{N}^{\circ}$ of buildings & 2835 & 2176 & $\mathbf{N}^{\circ}$ of buildings & 2172 & 2275 \\
\hline $48 \leq$ Lden $\leq 52.9$ & & & $45 \leq$ Lnight $\leq 49.9$ & & \\
\hline$\%$ of buildings & $17.1 \%$ & $13.2 \%$ & $\%$ of buildings & $13.1 \%$ & $13.8 \%$ \\
\hline $48 \leq$ Lden $\leq 52.9$ & & & $45 \leq$ Lnight $\leq 49.9$ & & \\
\hline $\mathbf{N}^{\circ}$ of buildings & 2902 & 2576 & $\mathbf{N}^{\circ}$ of buildings & 3045 & 2145 \\
\hline $43 \leq$ Lden $\leq 47.9$ & & & $40 \leq$ Lden $\leq 44.9$ & & \\
\hline$\%$ of buildings & $17.5 \%$ & $15.6 \%$ & $\%$ of buildings & $18.4 \%$ & $13 \%$ \\
\hline $43 \leq$ Lden $\leq 47.9$ & & & $40 \leq$ Lnight $\leq 44.9$ & & \\
\hline $\mathbf{N}^{\circ}$ of buildings & 1450 & 4980 & $\mathbf{N}^{\circ}$ of buildings & 4016 & 7674 \\
\hline Lden $\leq 42.9 \mathrm{~dB}(\mathrm{~A})$ & & & Lnight $\leq 39.9 \mathrm{~dB}(\mathrm{~A})$ & & \\
\hline$\%$ of buildings & $8.8 \%$ & $30.1 \%$ & $\%$ of buildings & $24.3 \%$ & $46.4 \%$ \\
\hline Lden $\leq 42.9 \mathrm{~dB}(\mathrm{~A})$ & & & Lnight $\leq 39.9 \mathrm{~dB}(\mathrm{~A})$ & & \\
\hline
\end{tabular}

These results underline once more the magnitude of the event.

It is important to note that, from the urban planning point of view, the area under investigation is characterized by residential buildings positioned at close distances from the main road source. The buildings (mainly blocks of flats) always have a facade directly facing the road and, in most cases, they have a quiet internal side (not exposed to traffic noise).
The analysis of the results shows that in large urban areas, such as the one under study, even in the period of lockdown (i.e. with a significant reduction in traffic volume) the RTN thresholds defined by the WHO Environmental Noise Guidelines are hardly respected.

To be noted that the results presented in this chapter are preliminary. In particular, the condition of applicability of DYNAMAP's prediction algorithm will need to be vali- 
dated by checking the membership "stability" of each noise monitoring stations to the assigned group.

\section{Conclusions}

March and April 2020 were unprecedented months as the entry into force of the restrictive regulation brought to a lockdown on 10 March 2020 The resulting prohibition of all non-essential commercial activities, businesses and industries amplified the social and economic ramifications of the COVID-19 pandemic throughout. The presence of a noise monitoring network in the urban area of Milan, developed in the framework of DYNAMAP project, allowed capturing the sound environment variation in the pre, during and post lockdown periods. The comparison with the same periods of 2019 brought to quantify a drop of noise levels between 5 and $7 \mathrm{~dB}$ in all monitored sites. This decrease is mainly due to the noise generated by road traffic: as regards the other noise sources (including those of natural origin), thanks to the ANED system implemented in the DYNAMAP project, it was, indeed, possible to verify that the differences between the two periods were less. During lockdown an increase of $14.9 \%$ and $16.4 \%$ of the exposed population to noises with Lden below $53 \mathrm{~dB}(\mathrm{~A})$ and Lnight below $45 \mathrm{~dB}(\mathrm{~A})$ with respect to 2019 , has been estimated. Data also highlight panic buying which prompted exceptional sales growth in fast-moving consumer goods as a reaction to stress/fear-induced restriction measures. The presented data are, however, preliminary and the conditions of applicability of DYNAMAP's prediction algorithm will need to be statistically validated by checking the membership "stability" of each noise monitoring stations to the assigned group. This work remains to be done.

Funding information: The authors state no funding involved.

Author contributions: All authors have accepted responsibility for the entire content of this manuscript and approved its submission.

Conflict of interest: The authors state no conflict of interest.

\section{References}

[1] Manzano JV, Almagro Pastor JA, Quesada RG, Aletta F, Oberman T et al. The "sound of silence" in Granada during the COVID-19 lockdown. Noise Mapp. 2021;8:16-31.

[2] Vogiatzis K, Zafiropoulou V, Gerolymatou G, Dimitriou D, Halkias B, Papadimitriou A, Konstantinidis A The noise climate at the time of SARS-CoV-2 VIRUS/COVID-19 disease in Athens - Greece: The case of Athens International Airport and the Athens Ring Road (Attiki Odos). Noise Mapp. 2020;7:154-170.

[3] Bartalucci C, Borchi F, Carfagni M Noise monitoring in Monza (Italy) during COVID-19 pandemic by means of the smart network of sensors developed in the LIFE MONZA project. Noise Mapp. 2020;7:199-211.

[4] Alsina-Pagès RM, Alías F, Bellucci P, Cartolano PP, Coppa I, Peruzzi L, Bisceglie A, Zambon G Noise at the time of COVID 19: The impact in some areas in Rome and Milan, Italy. Noise Mapp. 2020;7:248-264.

[5] Aletta F, Oberman T, Mitchell A, Tong H, Kang J Assessing the changing urban sound environment during the COVID-19 lockdown period using short-term acoustic measurements. Noise Mapp. 2020;7:123-134.

[6] Aletta F, Brinchi S, Carrese S, Gemma A, Guattari C, Mannini L, Patella SM. Analysing urban traffic volumes and mapping noise emissions in Rome (Italy) in the context of containment measures for the COVID-19 disease. Noise Mapp.2020;7:114-122.

[7] Sakagami K. A note on the acoustic environment in a usually quiet residential area after the 'state of emergency' declaration due to COVID-19 pandemic in Japan was lifted: supplementary survey results in post-emergency situations. Noise Mapp. 2020;7:192198.

[8] http://www.life-dynamap.eu/ [last accessed on 05/02/2021]

[9] Benocci R, Confalonieri C, Roman HE, Angelini A, Zambon G. Accuracy of the Dynamic Acoustic Map in a Large City Generated by Fixed Monitoring Units, Sensors. 2020;20(2).

[10] Benocci R, Bellucci P, Peruzzi L, Bisceglie A, Angelini F, Confalonieri $\mathrm{C}$, Zambon $\mathrm{G}$ Dynamic noise mapping in the suburban area of Rome (Italy). Environments 2019;6:79.

[11] Alsina-Pagès RM, Orga F, Alías F, Zambon G, Socoró JC, Benocci R. Anomalous events removal for automated traffic noise maps generation. Appl. Acoust. 2019;151:183-192.

[12] Orga F, Socoró JC, Alías F, Alsina-Pagès RM, Zambon G, Benocci $R$ et al. A Anomalous noise events considerations for the computation of road traffic noise levels: The DYNAMAP's Milan case study. 24th Int Congr Sound Vibr, ICSV; 2017.

[13] https://www.euro.who.int/en/publications/abstracts/environm ental-noise-guidelines-for-the-european-region-2018 [accessed on $05 / 02 / 2021]$ 